\title{
Stability of plane waves on deep water with dissipation
}

\author{
Nathan E. Canney, John D. Carter* \\ Seattle University \\ Mathematics Department \\ Seattle, WA 98122, USA
}

\begin{abstract}
The Benjamin-Feir modulational instability effects the evolution of perturbed planewave solutions of the cubic nonlinear Schrödinger equation (NLS), the modified NLS, and the band-modified NLS. Recent work demonstrates that the BenjaminFeir instability in NLS is "stabilized" when a linear term representing dissipation is added. In this paper, we add a linear term representing dissipation to the modified NLS and band-modified NLS equations and establish that the plane-wave solutions of these equations are linearly stable. Although the plane-wave solutions are stable, some perturbations grow for a finite period of time. We analytically bound this growth and present approximate time-dependent regions of wave-number space that correspond to perturbations that have increasing amplitudes.
\end{abstract}

Key words: Benjamin-Feir, dissipation, plane waves, NLS, stability

\section{Introduction}

The equations that describe the irrotational motion of an inviscid, incompressible, homogeneous fluid with a free surface are well known (see for example Landau \& Lifshitz, 1997). Let $\phi(\xi, \eta, \nu, \tau)$ denote the velocity potential of such a fluid resting on an impenetrable flat bed and let $\zeta(\xi, \eta, \tau)$ denote its surface displacement. If gravity is the only external force acting on the fluid, then the governing equations are

\footnotetext{
* Corresponding author.

Email addresses: canneyn@seattleu.edu (Nathan E. Canney), carterj1@seattleu.edu (John D. Carter).
} 


$$
\begin{aligned}
& \phi_{\nu}=\zeta_{\tau}+\phi_{\xi} \zeta_{\xi}+\phi_{\eta} \zeta_{\eta} \quad \text { at } \quad \nu=\zeta(\xi, \eta, \tau) \text {, } \\
& \phi_{\tau}+\frac{1}{2}|\nabla \phi|^{2}+g \zeta=0 \quad \text { at } \quad \nu=\zeta(\xi, \eta, \tau) \text {, } \\
& \nabla^{2} \phi=0 \quad \text { for } \quad-h<\nu<\zeta(\xi, \eta, \tau), \\
& \phi_{\nu}=0 \quad \text { at } \quad \nu=-h \text {. }
\end{aligned}
$$

Here $\nabla=(\partial / \partial \xi, \partial / \partial \eta, \partial / \partial \nu), g$ is the acceleration due to gravity, $h$ represents the depth of the undisturbed water above a horizontal and impermeable bed, $\nu$ is the vertical spatial coordinate, $\xi$ and $\eta$ are horizontal spatial coordinates and $\tau$ is time. All variables in (1) have physical dimensions.

Let $(k, l)$ and $a$ represent the characteristic wave number and characteristic amplitude of a packet of nearly monochromatic waves of small amplitude, respectively. Let $\delta k$ represent a small perturbation in $k$, let $\kappa^{2}=k^{2}+l^{2}$ and let $\epsilon$ be a small positive real number such that $\epsilon \ll 1$. Assuming small amplitude waves, $\kappa a=\mathcal{O}(\epsilon)$; nearly one-dimensional waves, $|l| / k=\mathcal{O}(\epsilon)$; slowly varying modulations, $\delta k / k=\mathcal{O}(\epsilon)$; and infinitely deep water, $h=\infty$; (1) can be asymptotically approximated through $\mathcal{O}\left(\epsilon^{3}\right)$ by the two-dimensional cubic nonlinear Schrödinger equation (NLS),

$$
i A_{t}+\frac{i}{2} A_{x}-\frac{1}{8} A_{x x}+\frac{1}{4} A_{y y}-\frac{1}{2}|A|^{2} A=0 .
$$

Here $A=A(x, y, t)$ is a complex-valued function that represents the envelope of the carrier wave of the fluid, $x$ and $y$ are horizontal spatial coordinates and $t$ is time. All variables in (2) have been rescaled from (1) and are dimensionless. Note that equation (2) is presented in a fixed reference frame, not the moving coordinate frame that is typically used. Complete details of the derivation of NLS can be found in Benney \& Roskes (1969), Davey \& Stewartson (1974) and Djordjevic \& Redekopp (1977).

Physical experiments conducted by Lake et al. (1977) demonstrate that NLS in one spatial dimension (equation $(2)$ with $\partial_{y}=0$ ) provides a qualitatively accurate description of the long-time evolution of wave packets for modulations corresponding to $\epsilon<0.1$. Lo \& Mei (1985) showed that for small amplitude waves, $\epsilon<0.1$, numerical predictions obtained from NLS in one dimension compare favorably with the exact calculations of Longuet-Higgins (1978). Although NLS works well in these situations, it is not the ideal model due to a number of limitations. These limitations include the fact that all of its experimental predictions become less reliable as $\epsilon$ increases (see for example Lo \& Mei, 1985) and that numerical simulations of NLS demonstrate energy leakage (see for example Martin \& Yuen, 1980; Yuen \& Lake, 1980).

In order to obtain an approximation of (1) that is valid for a wider range of $\epsilon$ than NLS, Dysthe (1979) carried out the NLS asymptotic analysis through 
$\mathcal{O}\left(\epsilon^{4}\right)$. Lo \& Mei (1987) generalized this work to allow for finite, but deep water, $(k h)^{-1}=\mathcal{O}(\epsilon)$, and obtained a system of equations named the modified nonlinear Schrödinger equations (MNLS),

$$
\begin{aligned}
& i A_{t}+\frac{i}{2} A_{x}-\frac{1}{8} A_{x x}+\frac{1}{4} A_{y y}-\frac{1}{2}|A|^{2} A+\frac{3 i}{8} A_{x y y}-\frac{i}{16} A_{x x x}+\frac{3 i}{2}|A|^{2} A_{x} \\
& -\frac{i}{4} A^{2} A_{x}^{*}-A \bar{\phi}_{x}=0 \quad \text { at } \quad z=0, \\
& \bar{\phi}_{z}=\frac{1}{2}\left(|A|^{2}\right)_{x} \quad \text { at } \quad z=0, \\
& \nabla^{2} \bar{\phi}=0 \quad \text { for } \quad-h<z<0 \text {, } \\
& \bar{\phi}_{z}=0 \quad \text { at } \quad z=-h .
\end{aligned}
$$

Here the variables $x, y, t$, and $A$ have the same meaning as in NLS, $\bar{\phi}=$ $\bar{\phi}(x, y, z, t)$ is a real-valued function representing the mean flow brought on by the radiation stress of the wave, $h$ represents the depth of the undisturbed water and $z$ is the vertical spatial coordinate. All variables in (3) are dimensionless and have been rescaled from (1) in the same manner as in NLS.

Lo \& Mei (1985) demonstrate that numerical simulations of MNLS in one spatial dimension compare more favorably with the physical experiments of $\mathrm{Su}$ (1982) than do numerical solutions of NLS if $\epsilon<0.15$. Although MNLS is a higher-order asymptotic approximation of (1) and provides better agreement with some physical experiments than NLS, it is still limited by the fact that it is only valid for slowly varying modulations, i.e. $\delta k / k=\mathcal{O}(\epsilon)$.

In order to create a model that accurately approximates (1) for a wider bandwidth of modulations, Trulsen \& Dysthe (1996) generalized the asymptotic analysis used in the derivation of (2) and (3). In their analysis, they allowed for modulations of $\mathcal{O}\left(\epsilon^{1 / 2}\right), \delta k / k=\mathcal{O}\left(\epsilon^{1 / 2}\right)$; and deep water, $(k h)^{-1}=$ $\mathcal{O}\left(\epsilon^{1 / 2}\right)$. They carried out the perturbation analysis through $\mathcal{O}\left(\epsilon^{7 / 2}\right)$ and derived the following system of equations they named the band-modified nonlinear Schrödinger equations (BMNLS)

$$
\begin{array}{rlrl}
i A_{t}+\frac{i}{2} A_{x}-\frac{1}{8} A_{x x}+\frac{1}{4} A_{y y}-\frac{1}{2}|A|^{2} A+\frac{3 i}{8} A_{x y y}-\frac{i}{16} A_{x x x}+\frac{3 i}{2}|A|^{2} A_{x} \\
-\frac{i}{4} A^{2} A_{x}^{*}-A \bar{\phi}_{x}+\frac{5}{128} A_{x x x x}-\frac{15}{32} A_{x x y y}+\frac{3}{32} A_{y y y y}+\frac{7 i}{256} A_{x x x x x} \\
-\frac{35 i}{64} A_{x x x y y}+\frac{21 i}{64} A_{x y y y}=0 & \text { at } \quad z=0, \\
\bar{\phi}_{z}=\frac{1}{2}\left(|A|^{2}\right)_{x} & \text { at } & z=0, \\
\nabla^{2} \bar{\phi}=0 & \text { for } & -h<z<0, \\
\bar{\phi}_{z}=0 & \text { at } & z & =-h .
\end{array}
$$


All of the variables in (4) have the same meaning as they do in (3) and are dimensionless. Comparisons between numerical simulations of BMNLS and physical experiments have been conducted by Trulsen et al. (1999).

Benjamin \& Feir (1967) established that the plane-wave solutions of (1) are unstable. Physically, they established that a uniform wave train of plane waves of moderate amplitude in deep water without dissipation is unstable with respect to small perturbations that consist of waves traveling in the same direction with nearly the same frequency. Zakharov (1968) derived the onedimensional cubic nonlinear Schrödinger equation and established that all of its plane-wave solutions are linearly unstable. Dysthe (1979) and Trulsen \& Dysthe (1996), respectively, established that all plane-wave solutions of the MNLS and BMNLS equations are linearly unstable.

These theories suggest that real-world plane waves of moderate amplitude on deep water are unstable. However, recent physical experiments conducted by Hammack \& Henderson (2003) and Hammack et al. (2005) have shown some apparently stable patterns of waves of moderate amplitude in deep water. One possible explanation for the discrepancy between the theory and experiments is that the mathematical models included above are not accurate because dissipation plays a role in the evolution of these waves. (None of the systems introduced above accounts for dissipation.)

In this paper, we assume that dissipation plays a role in the evolution of plane waves of moderate amplitude in deep water. We add a linear term representing dissipation to MNLS and BMNLS in order to model the effects of weak dissipation. We refer to these two new equations as DMNLS and DBMNLS respectively. There are two main results obtained in this paper.

(1) We establish that all plane-wave solutions of DMNLS and DBMNLS are linearly stable. This is a generalization of one of the results in Segur et al. (2005). (See Section 3.1.)

(2) We present approximate, time-dependent regions of wave-number space that admit perturbations with increasing amplitudes. (See Section 3.2.) 


\section{Dissipative BMNLS}

In order to examine the effect of weak dissipation, we consider the following generalization of (2)-(4)

$$
\begin{aligned}
& i A_{t}+\frac{i}{2} A_{x}-\frac{1}{8} A_{x x}+\frac{1}{4} A_{y y}-\frac{1}{2}|A|^{2} A \\
&+\rho_{1}\left(\frac{3 i}{8} A_{x y y}-\frac{i}{16} A_{x x x}+\frac{3 i}{2}|A|^{2} A_{x}-\frac{i}{4} A^{2} A_{x}^{*}-A \bar{\phi}_{x}\right) \\
&+\rho_{2}\left(\frac{5}{128} A_{x x x x}-\frac{15}{32} A_{x x y y}+\frac{3}{32} A_{y y y y}+\frac{7 i}{256} A_{x x x x x}-\frac{35 i}{64} A_{x x x y y}\right. \\
&\left.+\frac{21 i}{64} A_{x y y y}\right)+i \delta A=0, \quad \text { at } \quad z=0, \\
& \bar{\phi}_{z}=\frac{1}{2}\left(|A|^{2}\right)_{x} \quad \text { at } \quad \text { for } \quad-h<z<0, \\
& \nabla^{2} \bar{\phi}=0 \quad \text { at } \quad z=-h, \\
& \bar{\phi}_{z}=0 \quad z=0,
\end{aligned}
$$

where $\delta$ is a nonnegative constant representing dissipation. The parameters $\rho_{1}$ and $\rho_{2}$ have value 0 or 1 and are introduced to distinguish between terms that appear in NLS, MNLS and BMNLS. If $\delta=0$, then (5) reduces to one of (2), (3) or (4) depending on the values of $\rho_{1}$ and $\rho_{2}$. Segur et al. (2005) demonstrated that NLS with a linear term representing dissipation added (i.e. (5) with $\rho_{1}=\rho_{2}=0$ ) provides excellent agreement with observations from physical experiments of waves of small to moderate amplitude on deep water. This agreement was far superior than that obtained from any of the nondissipative models examined. Dissipation of this form in NLS has been examined by Miles (1967), Lake et al. (1977) and Mei \& Hancock (2003). It is important to note that this is one possible model of dissipation and that many other models exist including those of Trulsen \& Dysthe (1990), Hara \& Mei (1994), Shemer \& Chamesse (1999), Joseph \& Wang (2004) and Bridges \& Dias (2004).

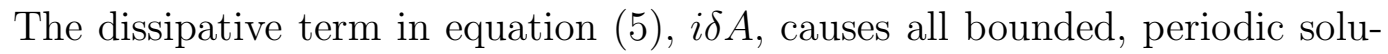
tions to decay to zero in time. This decay can be factored out via the following change of variables,

$$
\begin{aligned}
& A=\mathrm{e}^{-\delta t} \psi, \\
& \bar{\phi}=\mathrm{e}^{-2 \delta t} \phi,
\end{aligned}
$$

where $\psi=\psi(x, y, t)$ is a complex-valued function and $\phi=\phi(x, y, z, t)$ is a real-valued function. Substituting (6) into (5) and simplifying leads to the 
dissipative band-modified nonlinear Schrödinger equations (DBMNLS)

$$
\begin{gathered}
i \psi_{t}+\frac{i}{2} \psi_{x}-\frac{1}{8} \psi_{x x}+\frac{1}{4} \psi_{y y}-\frac{1}{2} \mathrm{e}^{-2 \delta t}|\psi|^{2} \psi, \\
+\rho_{1}\left(\frac{3 i}{8} \psi_{x y y}-\frac{i}{16} \psi_{x x x}+\mathrm{e}^{-2 \delta t}\left(\frac{3 i}{2}|\psi|^{2} \psi_{x}-\frac{i}{4} \psi^{2} \psi_{x}^{*}-\left.\psi \phi_{x}\right|_{z=0}\right)\right) \\
+\rho_{2}\left(\frac{5}{128} \psi_{x x x x}-\frac{15}{32} \psi_{x x y y}+\frac{3}{32} \psi_{y y y y}+\frac{7 i}{256} \psi_{x x x x x}-\frac{35 i}{64} \psi_{x x x y y}+\frac{21 i}{64} \psi_{x y y y y}\right) \\
\phi_{z}=\frac{1}{2}\left(|\psi|^{2}\right)_{x} \quad \text { at } \quad \text { for } \quad-h<z<0, \\
\nabla^{2} \phi=0 \quad \text { at } \quad z=-h .
\end{gathered}
$$

Unlike (5), this system of equations conserves the $\mathcal{L}_{2}$-norm,

$$
\mathcal{L}_{2}(\psi)=\int_{0}^{L_{y}} \int_{0}^{L_{x}}|\psi|^{2} d x d y
$$

in time. Here $L_{x}$ and $L_{y}$ are the periods of $\psi$ in the $x$ - and $y$-dimensions respectively. This conservation establishes that the solutions of (7) do not decay to zero.

In the deep-water limit $(h=\infty)$, the spatially-independent plane-wave solutions of DBMNLS are given by

$$
\begin{gathered}
\psi(t)=\psi_{0} \exp \left(-\frac{i \psi_{0}^{2}}{4 \delta}\left(1-\mathrm{e}^{-2 \delta t}\right)\right), \\
\phi=0,
\end{gathered}
$$

where $\psi_{0}$ is a real constant. Note that these solutions were chosen so that as $\delta \rightarrow 0$, they limit to the spatially-independent plane-wave solutions of NLS, MNLS and BMNLS,

$$
\psi(t)=\psi_{0} \exp \left(-\frac{i}{2} \psi_{0}^{2} t\right)
$$

Again, note that these solutions do not decay to zero.

\section{Linear stability analysis}

A solution $\psi(x, y, t)$ of $(7)$ is said to be stable if for every $\epsilon>0$ there exists a $\Delta>0$ such that if

$$
\left\|\psi_{p}(x, y, 0)-\psi(x, y, 0)\right\|^{2}<\Delta
$$

then

$$
\left\|\psi_{p}(x, y, t)-\psi(x, y, t)\right\|^{2}<\epsilon \quad \text { for all } t>0
$$


where $\psi_{p}$ and $\psi$ are governed by (7).

In order to study the linear stability of solutions of the form given in (9), consider perturbed solutions of the form

$$
\begin{gathered}
\psi_{p}(x, y, t)=\left(\psi_{0}+\epsilon u(x, y, t)+i \epsilon v(x, y, t)+\mathcal{O}\left(\epsilon^{2}\right)\right) \exp \left(-\frac{i \psi_{0}^{2}}{4 \delta}\left(1-\mathrm{e}^{-2 \delta t}\right)\right), \\
\phi_{p}(x, y, z, t)=0+\epsilon p(x, y, z, t)+\mathcal{O}\left(\epsilon^{2}\right)
\end{gathered}
$$

where $\epsilon$ is a small real parameter, and $u(x, y, t), v(x, y, t)$, and $p(x, y, z, t)$ are real-valued functions. The $\epsilon$ used in equation (13) has no relation to the $\epsilon$ used in the derivation of DBMNLS. Substituting (13) into (7), linearizing and separating into real and imaginary parts gives

$$
\begin{aligned}
& -v_{t}-\frac{1}{2} v_{x}-\frac{1}{8} u_{x x}+\frac{1}{4} u_{y y}+\mathrm{e}^{-2 \delta t}\left(-\psi_{0}^{2} u\right) \\
& +\rho_{1}\left(-\frac{3}{8} v_{x y y}+\frac{1}{16} v_{x x x}+\mathrm{e}^{-2 \delta t}\left(-\frac{7 \psi_{0}^{2}}{4} v_{x}-\left.\psi_{0} p_{x}\right|_{z=0}\right)\right) \\
& +\rho_{2}\left(\frac{3}{32} u_{y y y y}-\frac{21}{64} v_{x y y y y}-\frac{15}{32} u_{x x y y}+\frac{35}{64} v_{x x x y y}+\frac{5}{128} u_{x x x x}\right. \\
& \left.-\frac{7}{256} v_{x x x x x}\right)=0 \\
& u_{t}+\frac{1}{2} u_{x}-\frac{1}{8} v_{x x}+\frac{1}{4} v_{y y}+\rho_{1}\left(\frac{3}{8} u_{x y y}-\frac{1}{16} u_{x x x}+\mathrm{e}^{-2 \delta t}\left(\frac{5 \psi_{0}^{2}}{4} u_{x}\right)\right) \\
& +\rho_{2}\left(\frac{3}{32} v_{y y y y}+\frac{21}{64} u_{x y y y y}-\frac{15}{32} v_{x x y y}-\frac{35}{64} u_{x x x y y}+\frac{5}{128} v_{x x x x}\right. \\
& \left.+\frac{7}{256} u_{x x x x x}\right)=0, \\
& p_{z}=\psi_{0} u_{x} \quad \text { at } \quad z=0, \\
& \nabla^{2} p=0 \quad \text { for } \quad-\infty<z<0 \text {, } \\
& p_{z}=0 \quad \text { at } \quad z=-\infty \text {. }
\end{aligned}
$$

Without loss of generality, assume

$$
\begin{gathered}
u(x, y, t)=U(t) \mathrm{e}^{i m x+i n y}+c . c . \\
v(x, y, t)=V(t) \mathrm{e}^{i m x+i n y}+c . c . \\
p(x, y, z, t)=\frac{i m \psi_{0}}{\mu} U(t) \mathrm{e}^{i m x+i n y}+c . c .,
\end{gathered}
$$

where $m$ and $n$ are real parameters representing the wave numbers in the $x$ and $y$-dimensions respectively, $U$ and $V$ are complex-valued functions, $\mu=$ $\sqrt{m^{2}+n^{2}}$, and c.c denotes complex conjugate. Note that the definition of $p$ given in equation (15c) satisfies the restrictions given in equations (14c)-(14e). 
Substituting (15) into (14) leads to the following linear system for $U$ and $V$

$$
\left(\begin{array}{l}
U \\
V
\end{array}\right)^{\prime}=\mathbf{A}\left(\begin{array}{l}
U \\
V
\end{array}\right)+\mathrm{e}^{-2 \delta t} \mathbf{B}\left(\begin{array}{c}
U \\
V
\end{array}\right),
$$

where $\mathbf{A}$ and $\mathbf{B}$ are constant matrices given by

$$
\begin{gathered}
\mathbf{A}=\left(\begin{array}{cc}
a_{11} & a_{12} \\
-a_{12} & a_{11}
\end{array}\right), \\
\mathbf{B}=\left(\begin{array}{cc}
\rho_{1}\left(-\frac{5}{4} i m \psi_{0}^{2}\right) & 0 \\
\psi_{0}^{2}\left(-1+\rho_{1} \frac{m^{2}}{\mu}\right) & \rho_{1}\left(-\frac{7}{4} i m \psi_{0}^{2}\right)
\end{array}\right),
\end{gathered}
$$

where

$$
\begin{gathered}
a_{11}=-\frac{i}{2} m+\rho_{1}\left(-\frac{i}{16} m^{3}+\frac{3 i}{8} m n^{2}\right)+\rho_{2}\left(-\frac{7 i}{256} m^{5}+\frac{35 i}{64} m^{3} n^{2}-\frac{21 i}{64} m n^{4}\right), \\
a_{12}=-\frac{1}{8} m^{2}+\frac{1}{4} n^{2}+\rho_{2}\left(-\frac{5}{128} m^{4}+\frac{15}{32} m^{2} n^{2}-\frac{3}{32} n^{4}\right) .
\end{gathered}
$$

\subsection{A Bound on growth}

To our knowledge, an exact solution of the system given in equation (16) is not known. Since we are unable to determine $U$ and $V$ exactly, we establish a bound on their growth.

The eigenvalues and eigenvectors of the matrix $\mathbf{A}$ are given by

$$
\begin{gathered}
\lambda_{ \pm}=a_{11} \pm i a_{12} \\
\mathbf{v}_{+}=\left(\begin{array}{l}
1 \\
i
\end{array}\right), \quad \mathbf{v}_{-}=\left(\begin{array}{c}
1 \\
-i
\end{array}\right)
\end{gathered}
$$

Let

$$
\left(\begin{array}{c}
U \\
V
\end{array}\right)=\mathbf{T D}\left(\begin{array}{l}
y_{1} \\
y_{2}
\end{array}\right)
$$

where $y_{1}=y_{1}(t)$ and $y=y_{2}(t)$ are complex-valued functions, $\mathbf{T}$ is the matrix constructed from the eigenvectors of $\mathbf{A}, \mathbf{T}=\left(\mathbf{v}_{+} \mathbf{v}_{-}\right)$, and $\mathbf{D}$ is the diagonal matrix with $\exp \left(\lambda_{+} t\right)$ and $\exp \left(\lambda_{-} t\right)$ on the diagonal. Equation (21) establishes that $U$ and/or $V$ grow in $t$ if and only if $y_{1}$ and/or $y_{2}$ grow because the eigenvalues of $\mathbf{A}$ are purely imaginary. This change of variables allows (16) to be rewritten as

$$
\left(\begin{array}{l}
y_{1} \\
y_{2}
\end{array}\right)^{\prime}=\mathrm{e}^{-2 \delta t} \mathbf{D}^{-1} \mathbf{T}^{-1} \mathbf{B T D}\left(\begin{array}{l}
y_{1} \\
y_{2}
\end{array}\right) .
$$


Bounding everything on the right-hand-side of (22) gives

$$
\left(\begin{array}{l}
y_{1} \\
y_{2}
\end{array}\right)^{\prime} \leq \mathrm{e}^{-2 \delta t} \frac{\psi_{0}^{2}}{2}\left(1+\rho_{1}\left(3|m|+\frac{m^{2}}{\mu}\right)\right)\left(\begin{array}{ll}
1 & 1 \\
1 & 1
\end{array}\right)\left(\begin{array}{l}
y_{1} \\
y_{2}
\end{array}\right) \text {. }
$$

The method of successive approximations (see for example Ince, 1956) gives

$$
\left|y_{1}(t)\right|,\left|y_{2}(t)\right| \leq \sqrt{y_{1}(0)^{2}+y_{2}(0)^{2}} \exp \left(\frac{\psi_{0}^{2}}{2 \delta}\left(1+\rho_{1}\left(3|m|+\frac{m^{2}}{\mu}\right)\right)\left(1-\mathrm{e}^{-2 \delta t}\right)\right)
$$

Maximizing with respect to $t$ and transforming back to the original variables gives

$$
|U(t)|^{2}+|V(t)|^{2} \leq \sqrt{U(0)^{2}+V(0)^{2}} \exp \left(\frac{\psi_{0}^{2}}{\delta}\left(1+\rho_{1}\left(3|m|+\frac{m^{2}}{\mu}\right)\right)\right) .
$$

Linear stability is established for any $\delta>0$ because given any $\epsilon>0$, choosing

$$
\Delta=\epsilon / \exp \left(\frac{\psi_{0}^{2}}{\delta}\left(1+\rho_{1}\left(3|m|+\frac{m^{2}}{\mu}\right)\right)\right),
$$

ensures that (11)-(12) are satisfied.

\subsection{Approximate regions of growth}

Equation (16) can be rewritten as

$$
\left(\begin{array}{c}
U \\
V
\end{array}\right)^{\prime}=\mathbf{G}(t)\left(\begin{array}{l}
U \\
V
\end{array}\right)
$$

where $\mathbf{G}(t)=\mathbf{A}+\mathrm{e}^{-2 \delta t} \mathbf{B}$. Formally, the eigenvalues of $\mathbf{G}, \Lambda_{ \pm}(t)$, determine regions of $(m, n)$-space that correspond to perturbations that have increasing amplitude at a time $t$. If $\Lambda(t)$ has a positive real part at a time $t$, then the amplitude of the corresponding perturbation is growing at that time $t$. If $\Lambda(t)$ has zero real part, then the amplitude of the corresponding perturbation remains constant at that time $t$. If $\Lambda(t)$ has a negative real part, then the amplitude of the corresponding perturbation is decaying at that time $t$. These statements are exact if $\mathbf{G}$ is constant, but are only approximate in this situation because $\mathrm{G}$ is nonconstant.

The eigenvalues of $\mathbf{G}$ are given by

$$
\begin{aligned}
\Lambda_{ \pm}(t)= & a_{11}-\frac{3}{2} i \mathrm{e}^{-2 \delta t} m \rho_{1} \psi_{0}^{2} \\
& \pm \sqrt{-a_{12}^{2}-a_{12} \mathrm{e}^{-2 \delta t} \psi_{0}^{2}-\frac{1}{16} m^{2} \rho_{1}^{2} \psi_{0}^{4} \mathrm{e}^{-4 \delta t}+a_{12} \mathrm{e}^{-2 \delta t} m^{2} \rho_{1} \psi_{0}^{2} \frac{m^{2}}{\mu}}
\end{aligned}
$$



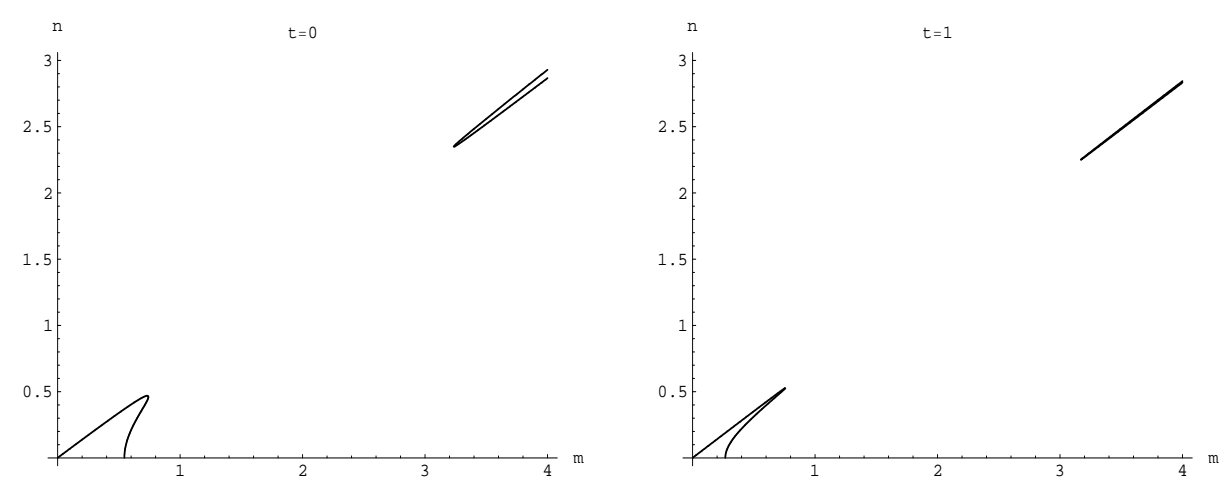

Fig. 1. Plots of $(m, n)$-space where $\operatorname{Re}(\Lambda(t))=0$ with $\delta=1$ and $\psi_{0}=0.3$ at $t=0$ and at $t=1$ for DMNLS.
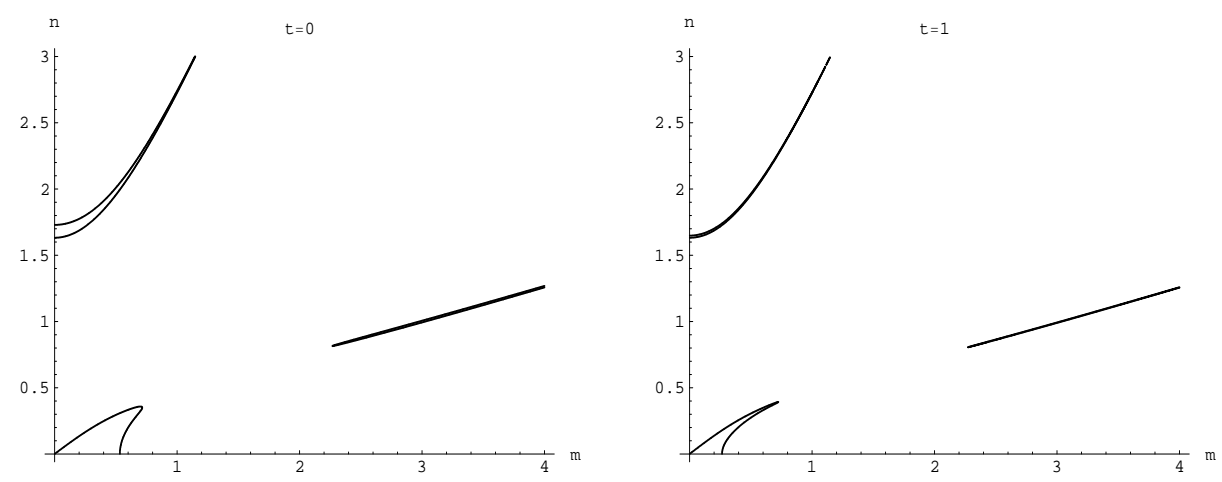

Fig. 2. Plots of $(m, n)$-space where $\operatorname{Re}(\Lambda(t))=0$ with $\delta=1$ and $\psi_{0}=0.3$ at $t=0$ and at $t=1$ for DBMNLS.

Figure 1 contains plots of $(m, n)$-space where $\operatorname{Re}(\Lambda(t))=0$ at $t=0$ and $t=1$ for DMNLS with $\delta=1$ and $\psi_{0}=0.3$. Figure 2 contains plots of $(m, n)$ space where $\operatorname{Re}(\Lambda(t))=0$ at $t=0$ and at $t=1$ for DBMNLS with $\delta=1$ and $\psi_{0}=0.3$. In each plot, the regions between the curves correspond to $\Lambda$ 's that have positive real part. The $t=0$ (zero dissipation) case of these (or equivalent) plots were obtained by Dysthe (1979), Trulsen \& Dysthe (1996) and Trulsen et al. (2000).

As demonstrated in these figures, the region of $(m, n)$-space corresponding to growing modes decreases in size between $t=0$ and $t=1$. This trend persists as $t$ increases and the curves continue to draw closer and closer to one another. There are two possibilities. If the point $(m, n)$ is initially outside of the curves, then the amplitude of the corresponding perturbation will not grow. If the point $(m, n)$ is initially between the curves, there is a time $t=t^{*}$ when one of the curves crosses through the point leaving it outside of the region. This establishes that the amplitude of the corresponding perturbation will grow for $t<t^{*}$ and will remain constant for $t>t^{*}$. Therefore, only finite growth is possible. 


\section{Conclusions}

- For any nonzero amount of linear dissipation (i.e. $\delta>0$ in equation (7)), the plane-wave solutions of DMNLS and DBMNLS are linearly stable.

- As $\delta \rightarrow 0$, the right-hand-side of (25) increases without bound. There is no bound on either $U$ or $V$ if $\delta=0$. This is consistent with the work of Dysthe (1979) and Trulsen \& Dysthe (1996) in which the plane-wave solutions of MNLS and BMNLS are shown to be linearly unstable.

- The amplitude of a perturbation may grow, possibly by a large amount. Regardless, linear stability is established because the growth is guaranteed to be finite.

- Similar computations can be applied to the generalization of NLS derived by Trulsen et al. (2000). For this problem, the bound in (25) limits the growth of any perturbation.

We acknowledge helpful discussions with Harvey Segur and Diane Henderson. This material is based upon work supported by the National Science Foundation under grant DMS-0139771.

\section{References}

Benjamin, T. \& FeiR, J. 1967 The disintegration of wave trains on deep water. Part 1. Journal of Fluid Mechanics 27 (3), 417-430.

Benney, D. \& Roskes, G. 1969 Wave instabilities. Studies in Applied Mathematics 48 (377).

Bridges, T. \& Dias, F. 2004 On the enhancement of the Benjamin-Feir instability due to dissipation. Submitted for publication .

Davey, A. \& Stewartson, K. 1974 On three-dimensional packets of surface waves. Proceedings of the Royal Society Series A 338, 101-110.

DJordjevic, V. \& ReDEKOPP, L. 1977 On two-dimensional packets of capillary-gravity waves. Journal of Fluid Mechanics 79 (4), 703-714.

Dysthe, K. 1979 Note on a modification of the nonlinear Schrödinger equation for application to deep water waves. Proceedings of the Royal Society London A 369, 105-114.

Hammack, J. \& Henderson, D. 2003 Experiments on deep-water waves with two-dimensional surface patterns. Journal of Offshore Mechanics and Arctic Engineering 125, 48-53.

Hammack, J., Henderson, D. \& Segur, H. 2005 Deep-water waves with persistant, two-dimensional surface patterns. Journal of Fluid Mechanics 532, 1-51.

HARA, T. \& MEI, C. 1994 Wind effects on the evolution of gravity-capillary waves. Journal of Fluid Mechanics 267, 221-250.

INCE 1956 Ordinary Differential Equations. Dover.

Joseph, D. \& WANG, J. 2004 The dissipation approximation and viscous potential flow. Journal of Fluid Mechanics 505, 365-377. 
Lake, B., Yuen, H., Rungaldier, H. \& Ferguson, W. 1977 Nonlinear deep-water waves: theory and experiment. Part 2. Evolution of a continuous wave train. Journal of Fluid Mechanics 83, 49-74.

Landau, L. \& Lifshitz, E. 1997 Fluid Mechanics. Butterworth Heinemann, Oxford.

Lo, E. \& MEI, C. 1985 A numerical study of water-wave modulation based on a higher-order nonlinear Schrödinger equation. Journal of Fluid Mechanics 150, 395-416.

Lo, E. \& MeI, C. 1987 Slow evolution of nonlinear deep water waves in two horizontal directions: A numerical study. Wave motion 9, 245-259.

Longuet-Higgins, M. 1978 The instabilities of gravity waves on deep water. II. Subharmonics. Proceedings of the Royal Society London A 360, 489-505.

Martin, D. \& Yuen, H. 1980 Quasi-recurring energy leakage in the twodimensional nonlinear Schrödinger equation. Physics of Fluids 23, 881-883.

Mei, C. \& HANCOCK, M. 2003 Weakly nonlinear surface waves over a random seabed. Journal of Fluid Mechanics 475, 247-268.

Miles, J. 1967 Surface-wave damping in closed basins. Proceedings of the Royal Society London A 297, 459-475.

Segur, H., Henderson, D., Carter, J., Hammack, J., Li, C., Pheiff, D. \& Socha, K. 2005 Stabilizing the Benjamin-Feir instability. Journal of Fluid Mechanics 539, 229-271.

Shemer, L. \& Chamesse, M. 1999 Experiments on nonlinear gravitycapillary waves. Journal of Fluid Mechanics 380, 205-232.

Su, M. 1982 Evolution of groups of gravity waves with moderate to high steepness. Physics of Fluids 25, 2167-2174.

Trulsen, K. \& Dysthe, K. 1990 Frequency down-shift through selfmodulation and breaking. In Water Wave Kinematics (ed. A. Torum \& O. Gudmestad), pp. 561-572. Kluwer.

Trulsen, K. \& Dysthe, K. 1996 A modified nonlinear Schrödinger equation for broader bandwidth gravity waves on deep water. Wave Motion 24, 281289.

Trulsen, K., Kliakhandler, I., Dysthe, K. \& Verlarde, M. 2000 On weakly nonlinear modulation of waves on deep water. Physics of Fluids 12, 2432-2437.

Trulsen, K., Stansberg, C. \& Verlarde, M. 1999 Laboratory evidence of three-dimensional frequency downshift of waves in a long tank. Physics of Fluids 11 (1), 235-237.

Yuen, H. \& Lake, B. 1980 Instabilities of waves on deep water. Annual Review of Fluid Mechanics 12, 303-334.

Zakharov, V. 1968 Stability of periodic waves of finite amplitude on the surface of a deep fluid. Soviet Physics Journal of Appl. Mech. Tech. Phys. 4,86 . 